\title{
Bounds on metric dimensions of graphs with edge disjoint cycles
}

\author{
Jelena Sedlar ${ }^{1}$, \\ Riste Škrekovski ${ }^{2,3}$ \\ ${ }^{1}$ University of Split, Faculty of civil engineering, architecture and geodesy, Croatia \\ ${ }^{2}$ University of Ljubljana, FMF, 1000 Ljubljana, Slovenia \\ ${ }^{3}$ Faculty of Information Studies, 8000 Novo Mesto, Slovenia
}

October 21, 2020

\begin{abstract}
In a graph $G$, cardinality of the smallest ordered set of vertices that distinguishes every element of $V(G)$ is the (vertex) metric dimension of $G$. Similarly, the cardinality of such a set is the edge metric dimension of $G$, if it distinguishes $E(G)$. In this paper these invariants are considered first for unicyclic graphs, and it is shown that the vertex and edge metric dimensions obtain values from two particular consecutive integers, which can be determined from the structure of the graph. In particular, as a consequence, we obtain that these two invariants can differ for at most one for a same unicyclic graph. Next we extend the results to graphs with edge disjoint cycles showing that the two invariants can differ at most by $c$, where $c$ is the number of cycles in such a graph. We conclude the paper with a conjecture that generalizes the previously mentioned consequences to graphs with prescribed cyclomatic number $c$ by claiming that the difference of the invariant is still bounded by $c$.
\end{abstract}

\section{Introduction}

Here we consider only simple and connected graphs. In a graph $G$, we denote by $d_{G}(u, v)$ (or simply $d(u, v)$ if no confusion arises) the distance between two vertices $u, v \in V(G)$. Now, if $d_{G}(u, s) \neq d_{G}(v, s)$, for some vertices $s, u, v$ of $G$, then we say that $s$ distinguishes (or resolves) $u$ and $v$. If any two vertices $u$ and $v$ are distinguished by at least one vertex of a subset $S \subseteq V(G)$, then we say that $S$ is a metric generator for $G$. The cardinality of the smallest metric generator is called the metric dimension of $G$, and it is denoted by $\operatorname{dim}(G)$. This notion for graphs was independently introduced by [3] and [11], under the names resolving sets and locating sets, respectively. Even before this notion was introduced for the realm of metric spaces [1]. In the paper, as we deal wih several types of 
metric dimension, in order to emphasize that we deal in certain situation with the usual metric dimension, we use the word "vertex" as a prefix, and say a vertex metric generator and a vertex metric dimension.

The concept of metric dimension was recently extended from resolving vertices to resolving edges of a graph by Kelenc, Tratnik and Yero [5]. Similarly as above, a vertex $s \in V(G)$ distinguishes two edges $e, f \in E(G)$ if $d_{G}(s, e) \neq d_{G}(s, f)$, where $d_{G}(e, s)=$ $d_{G}(u v, s)=\min \{d(u, s), d(v, s)\}$. A set of vertices $S \subseteq V(G)$ is an edge metric generator for $G$, if any two edges of $G$ are distinguished by a vertex of $S$. The cardinality of the smallest edge metric generator is called the edge metric dimension of $G$, and it is denoted by $\operatorname{edim}(G)$. In [5] it was shown that determining the edge metric dimension of a graph is NP-hard. Also for trees, grid graphs, wheels and some other graph classes are given bounds and closed formulas. In particular, families of graphs for which $\operatorname{dim}(G)<\operatorname{edim}(G)$, or $\operatorname{dim}(G)=\operatorname{edim}(G)$, or $\operatorname{dim}(G)>\operatorname{edim}(G)$ were presented.

Edge metric dimension immediately attracted big attention. In [9], Peterin and Yero were considering the edge metric dimension of corona, join and lexicographic products of graphs. In [13] was considered the maximum possible value of edge metric dimension amongst graphs of prescribed order. And, Zubrilina [14] showed that it is not posisble to bound the metric dimension of a graph $G$ by some function of the edge metric dimension of $G$.

For a wider and systematic introduction of the topic of metric dimension, we recommend the $\mathrm{PhD}$ thesis of Kelenc [4. All three works [4, 5, 6] propose various open problems and research directions for possible further work.

Throughout the paper we will use the following notation. The only cycle in the unicyclic graph $G$ that is under our consideration is denoted by $C=v_{0} v_{1} \cdots v_{g-1}$, where $g$ is the length of $C$ (i.e. $g=|V(C)|)$. The connected component of $G-E(C)$ containing vertex $v_{i}$ is denoted by $T_{v_{i}}$.

A thread in a graph $G$ is a path $u_{1} u_{2} \cdots u_{k}$ in which all vertices are of degree 2 except for $u_{k}$ which is of degree 1 and $u_{1}$ is a neighbour of a vertex $v \in V(G)$ with $\operatorname{deg}(v) \geq 3$. Note that vertices of the only cycle in a unicyclic can have acyclic structures attached to them by an edge. When such acyclic structure does not contain a vertex of degree $\geq 3$ then it is a thread, otherwise we call such structure a branch (for illustration see Figure 1). When a branch is attached to a vertex of the cycle, then there is certainly a pair of vertices and a pair of edges in the branch which are on the same distance from the cycle $C$ which, therefore, cannot be distinguished by a vertex from outside the branch. Note that the same branching phenomenon occurs when there are two (or more) threads attached to the same vertex of the cycle and no branches. This is of interest to us, so we introduce the following definition. For a vertex $v$ from a unicyclic graph $G$ we say that it is a branching vertex if $v \notin V(C)$ and $\operatorname{deg}(v) \geq 3$ or if $v \in V(C)$ and $\operatorname{deg}(v) \geq 4$. We say that a vertex $v_{i} \in V(C)$ is branch-active if $T_{v_{i}}$ contains a branching vertex. Note that a vertex $v_{i}$ from the cycle $C$ is branch-active if there is a branch hanging at $v_{i}$ but also if there is more than one thread attached to it. Denote by $b(C)$ the number of all branch-active vertices on $C$. As the cycle $C$ is the only cycle in a unicyclic graph, we can use notation $b(G)$ instead of $b(C)$.

We say that a subgraph $H$ of a graph $G$ is an isometric subgraph, if for any two vertices $u, v \in V(H)$ it holds that $d_{H}(u, v)=d_{G}(u, v)$. The following notation for paths is 


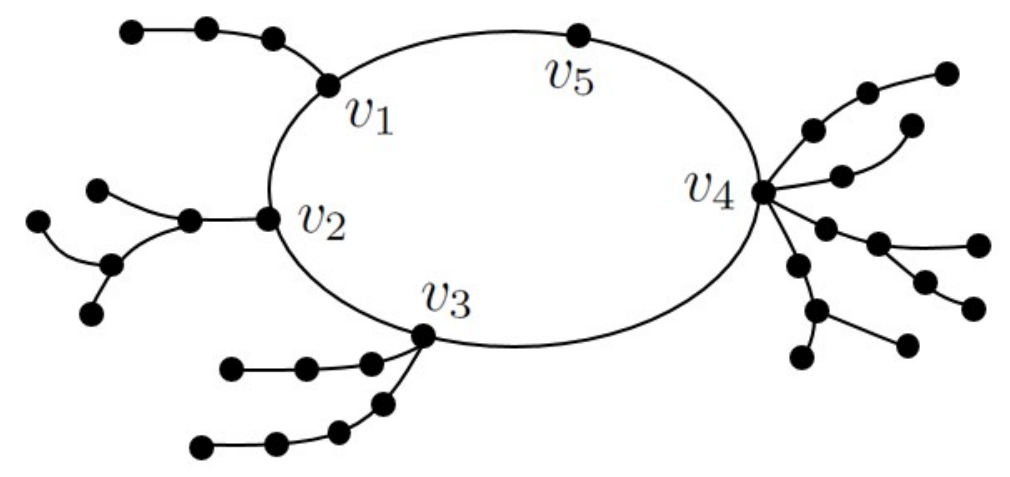

Figure 1: An illustration of threads and branches: $v_{1}$ has a thread attached and $v_{2}$ has a branch attached. Vertices of the cycle can have more than one acyclic structure attached to them ( $v_{3}$ has two threads attached and $v_{4}$ has two threads and two branches attached) or none (vertex $v_{5}$ ). Branch-active vertices are $v_{2}, v_{3}$ and $v_{4}$ (even though $v_{3}$ does not have a branch attached). Finally, $T_{v_{1}}$ and $T_{v_{2}}$ consist of a thread and a branch attached to a respective vertices, but $T_{v_{3}}$ consists of both threads attached to $v_{3}$ and the same holds for $T_{v_{4}}$ - it consists of all threads and branches attached to $v_{4}$. Finally, $T_{v_{5}}$ is trivial, it consists only from the vertex $v_{5}$.

used. Suppose that $P$ is a path and $u, v \in V(P)$, then by $P[u, v]$ we denote the subpath of $P$ connecting vertices $u$ and $v$, while by $P(u, v)$ we denote $P[u, v]-\{u, v\}$. Notions $P[u, v)$ and $P(u, v]$ are also used and they denote the subpaths where only one of the end-vertices of $P[u, v]$ is excluded. For any edge $e \in E(G)$, let $G / e$ be the graph obtained from $G$ by contracting $e$.

\section{Branch-resolving sets}

In the paper, we will establish lower and upper bounds on metric dimensions for unicyclic graphs. As for considering the lower bound, we define that a set $S \subseteq V(G)$ of a graph $G$ is branch-resolving if for every $v \in V(G)$ of degree at least 3, the set $S$ contains a vertex from all threads starting at vertex $v$ except possibly from one such thread. In this short section, we provide several properties of branch-resolving sets. Notice that one can always choose a branch-resolving set comprised of leaves.

Let us denote by $\ell(v)$ the number of all threads attached to a vertex $v$ of $G$, and let

$$
L(G)=\sum_{v \in V(G), \ell(v)>1}(\ell(v)-1)
$$

Note that for every branch-resolving set $S$ we have $|S| \geq L(G)$ with equality holding for branch-resolving sets of minimum cardinality. Regarding the trees, the following nice result is well known, see [5, 7]. 
Proposition 1 For every tree $T$ that is not a path, it holds

$$
\operatorname{dim}(T)=\operatorname{edim}(T)=L(T) .
$$

Let $G$ be a unicyclic graph, let $S \subseteq V(G)$ be a set of vertices in $G$, and let $v_{i}$ be a vertex from $C$. We say that a vertex $v_{i}$ is $S$-active, if $T_{v_{i}}$ contains a vertex from $S$. We will mainly be interested in $S$-active vertices with respect to a given branch-resolving set $S$. Let $A(S) \subseteq V(C)$ be the set of vertices in $V(C)$ which are $S$-active, also let $a(S)$ be the cardinality of the set $A(S)$.

Lemma 2 Let $S$ be a metric generator or an edge metric generator of a unicyclic graph $G$. Then $S$ is a branch-resolving set with $a(S) \geq 2$.

Proof. If $S$ is not a branch-resolving set, there is a vertex $v \in V(G)$ of degree at least three such that there are two threads $x_{1} x_{2} \cdots x_{k}$ and $y_{1} y_{2} \cdots y_{l}$ attached to $v$ (vertices $x_{1}$ and $y_{1}$ are adjacent to $v$ ) such that $S$ does not contain a vertex from these two threads. Let $G_{v}$ be the connected component of $G-\left\{v x_{1}, v y_{1}\right\}$ which contains $v$. Note that $S \subseteq V\left(G_{v}\right)$ and $d\left(x_{1}, z\right)=d\left(y_{1}, z\right)$ for every $z \in V\left(G_{v}\right)$. Therefore, $S$ does not distinguish $x_{1}$ and $y_{1}$, and so $S$ is not a metric generator. Also, note that $d\left(x_{1} v, z\right)=d\left(y_{1} v, z\right)$ for every $z \in V\left(G_{v}\right)$, which implies that $S$ does not distinguish edges $x_{1} v$ and $y_{1} v$, so neither is $S$ an edge metric generator. In both cases we obtain a contradicton.

If $a(S)=0$ then $S=\phi$ and the claim is obvious. If $a(S)=1$, then let $v_{i}$ be the vertex from cycle $C$ in $G$ that is $S$-active. Note that for the connected component $T_{v_{i}}$ it holds that $S \subseteq V\left(T_{v_{i}}\right)$. Let $v_{i-1}$ and $v_{i+1}$ be two neighbors of $v_{i}$ on $C$. Then, $v_{i-1}$ and $v_{i+1}$ are not distinguished by $S$, so $S$ is not a metric generator. Also, edges $v_{i-1} v_{i}$ and $v_{i} v_{i+1}$ are not distinguished by $S$, so $S$ is not an edge metric generator either.

Above result tells that every generating set is branch-resolving, the opposite does not hold but still by a branch-resolving set we can distinguish "local" pairs of vertices and edges as it is shown in the next lemma.

Lemma 3 Let $G$ be a unicyclic graph and $S \subseteq V(G)$ a branch-resolving set with $a(S) \geq 2$. Then, any two vertices (also any two edges) from a same connected component of $G-E(C)$ are distinguished by $S$.

Proof. Since $a(S) \geq 2$, there are at least two vertices on the cycle $C$ which are $S$-active, say $v_{i}$ and $v_{j}$. Let $x$ and $x^{\prime}$ be two vertices from a same component of $G-E(C)$, say $x$ and $x^{\prime}$ both belong to $T_{v_{k}}$. Without loss of generality, we may assume that $k \neq i$. Then $x$ and $x^{\prime}$ are distinguished by a vertex $s \in S \cap V\left(T_{v_{i}}\right)$ in all cases except when $d\left(x, v_{k}\right)=d\left(x^{\prime}, v_{k}\right)$. Therefore, suppose that $d\left(x, v_{k}\right)=d\left(x^{\prime}, v_{k}\right)$ and let $P$ be the only path in $G$ connecting vertices $x$ and $x^{\prime}$. Denote by $v$ the middle vertex of the path $P$ which must exist because of our assumption that $d\left(x, v_{k}\right)=d\left(x^{\prime}, v_{k}\right)$, and so $P$ is of even length. Let $G_{v}$ be the connected component of $G-E(P)$ containing vertex $v$. Note that $x$ and $x^{\prime}$ are distinguished by all vertices outside $G_{v}$. So, outside $G_{v}$ there cannot be any vertex from $S$.

Let $G_{x}$ and $G_{x^{\prime}}$ be the connected components of $G-v$ containing vertices $x$ and $x^{\prime}$ respectively. If both $G_{x}$ and $G_{x^{\prime}}$ are threads, then vertex $v$ has two threads attached to 
it which do not contain a vertex from $S$ which is a contradiction. Therefore, $G_{x}$ or $G_{x^{\prime}}$ is a branch i.e. it contains at least one vertex of degree $\geq 3$, say $G_{x}$ is a branch. Therefore, $G_{x}$ must contain at least two threads attached to the same vertex which do not contain a vertex from $S$ which is a contradiction.

Suppose now that $e=x y$ and $e^{\prime}=x^{\prime} y^{\prime}$ are two edges from the same $T_{v_{k}}$. We may assume that $x$ is closer to $v_{k}$ than $y$, and similarly $x^{\prime}$ is closer to $v_{k}$ than $y^{\prime}$. We may also assume $d\left(x, v_{k}\right)=d\left(x^{\prime}, v_{k}\right)$, otherwise $e$ and $e^{\prime}$ will be distinguished by any vertex $s \in S \backslash V\left(T_{v_{k}}\right)$. Now, let $P$ be the shortest path connecting vertices $x$ and $x^{\prime}$. Note that the fact that $d\left(x, v_{k}\right)=d\left(x^{\prime}, v_{k}\right)$ implies $P$ is of even length which means there is a vertex $v$ sitting in the middle of $P$ which is of degree at least 3. Note that $e$ and $e^{\prime}$ are distinguished by $S$ unless $S \subseteq V\left(G_{v}\right)$ where $G_{v}$ is the connected component of $G-E(P)$ containing vertex $v$. Suppose therefore that $S \subseteq V\left(G_{v}\right)$. Observe that connected components $G_{y}$ and $G_{y^{\prime}}$ of $G-v$ that contains $y$ and $y^{\prime}$, respectively, are different. If both $G_{y}$ and $G_{y^{\prime}}$ are threads, then there are two threads attached to $v$ which do not contain a vertex from $S$ which is a contradiction. Otherwise, $G_{y}$ or $G_{y^{\prime}}$ is a branch, then it must contain a vertex from $S$ and consequently $S \nsubseteq V\left(G_{v}\right)$, a contradiction.

\section{Geodesic triples}

Branch-resolving sets enable us to distinguish vertices and edges from same $T_{v_{i}}$ 's. Now, we introduce "small" sets which enable us to distinguish vertices and edges from distinct $T_{v_{i}}$ 's. Let $v_{i}, v_{j}$, and $v_{k}$ be three vertices belonging to the only cycle $C$ in a unicyclic graph $G$. We say that $v_{i}, v_{j}$, and $v_{k}$ form a geodesic triple of vertices on $C$, if

$$
d\left(v_{i}, v_{j}\right)+d\left(v_{j}, v_{k}\right)+d\left(v_{i}, v_{k}\right)=|V(C)| .
$$

Observe that for any two vertices of $C$, we can easily choose a thrid one such that they form a geodesic triple. Let us now proceed to prove that geodesic triple of vertices distinguishes all pairs of vertices and all pairs of edges which are not in the same connected component of $G-E(C)$. Notice that the edges of $C$ are also considered here.

Lemma 4 Let $G$ be a unicyclic graph and let $S$ be a geodesic triple of vertices from $C$. Then, $S$ distinguishes any two vertices that belong to two distinct components of $G-E(C)$.

Proof. Suppose to the contrary, i.e. $S$ does not distinguish vertices $x$ and $x^{\prime}$ that belong to distinct components of $G-E(C)$. We may assume that $x$ belongs to $T_{v_{i}}$ and $x^{\prime}$ belongs to $T_{v_{j}}$ with $i \neq j$. Let $v_{j}^{*}$ be the antipodal of $v_{j}$ such that in case when $g$ is odd, it has two antipodals, we choose the one closer to $v_{i}$. And similarly, let $v_{i}^{*}$ be the antipodal of $v_{i}$ that is closer to $v_{j}$. See Figure 2, where it is assumed that $C$ is odd, $v_{i}$ (resp. $v_{j}$ ) is connected to its two antipodal vertices, the one that is closer to $v_{j}$ (resp. $v_{i}$ ) is denoted by $v_{i}^{*}\left(\operatorname{resp} . v_{j}^{*}\right)$.

Let $P_{1}$ and $P_{2}$ be the two paths in $G$ connecting $x$ and $x^{\prime}$. If $P_{1}$ and $P_{2}$ are of different length, we assume that $P_{1}$ is shorter than $P_{2}$. Thus, $v_{i}^{*}$ and $v_{j}^{*}$ belong to $P_{2}$. In what follows, we show several claims regarding $P_{1}, P_{2}$, and $S$ in order to complete the proof. 


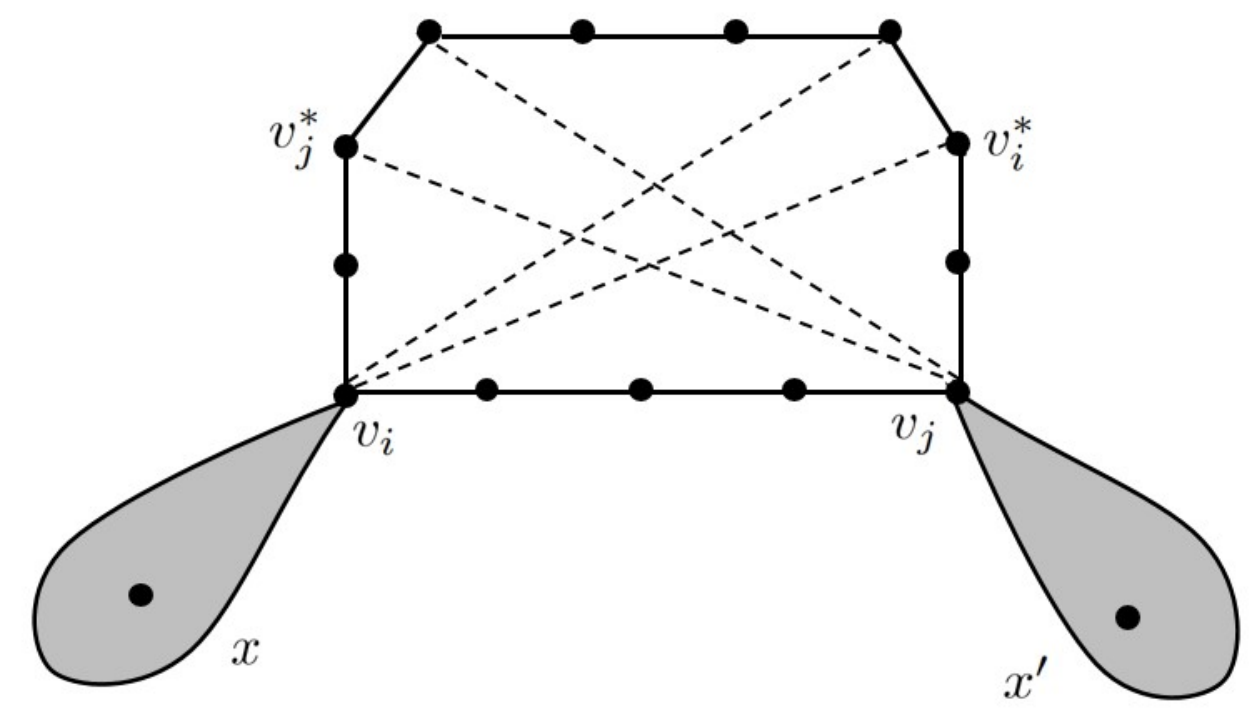

Figure 2: An illustration of the situation in Lemma 4 with $C$ being of odd length: vertex $v_{i}$ has two antipodal vertices where we choose for $v_{i}^{*}$ the one of the two closer to $v_{j}$ and the same holds for $v_{j}$.

Claim 1. Each of $P_{1}\left[v_{i}, v_{j}\right]$ and $P_{2}\left(v_{i}^{*}, v_{j}^{*}\right)$ contains at most one vertex from $S$.

Regarding $P_{1}\left[v_{i}, v_{j}\right]$, this is due to the fact $P_{1}$ is a shortest path between $x$ and $x^{\prime}$ which implies it is an isometric path. Now, the claim follows from the fact that an isometric path cannot contain two distinct vertices on equal distance to one of its end-vertices.

Suppose now that $P_{2}\left(v_{i}^{*}, v_{j}^{*}\right)$ contains at least two vertices from $S$, say $s_{1}$ and $s_{2}$. The fact that $s_{\ell}(\ell \in\{1,2\})$ does not distinguish $x$ and $x^{\prime}$ implies

$$
d\left(s_{\ell}, v_{i}\right)+d\left(v_{i}, x\right)=d\left(s_{\ell}, v_{j}\right)+d\left(v_{j}, x^{\prime}\right),
$$

or equivalently

$$
d\left(s_{\ell}, v_{i}\right)-d\left(s_{\ell}, v_{j}\right)=d\left(v_{j}, x^{\prime}\right)-d\left(v_{i}, x\right) .
$$

As the right side of the equality is the same for both $\ell=1$ and $\ell=2$, we promptly derive that

$$
d\left(s_{1}, v_{i}\right)-d\left(s_{1}, v_{j}\right)=d\left(s_{2}, v_{i}\right)-d\left(s_{2}, v_{j}\right) .
$$

But this is not possible due to the fact that $P_{2}\left[v_{j}, v_{j}^{*}\right]$ and $P_{2}\left[v_{i}, v_{i}^{*}\right]$ are isometric paths, and all these four distances are realized by subpaths of $P_{2}$. This establishes the claim.

Claim 2. If $P_{2}\left[v_{i}, v_{j}^{*}\right]$ contains a vertex from $S$ then $P_{2}\left(v_{j}^{*}, v_{j}\right]$ contains no vertex from $S$. Suppose the claim is false and $s_{1}$ is in $P_{2}\left[v_{i}, v_{j}^{*}\right]$ and $s_{2}$ is in $P\left(v_{j}^{*}, v_{j}\right]$. Since $s_{1}$ does not distinguish $x$ and $x^{\prime}$, it follows that $d\left(v_{i}, x\right)=d\left(v_{i}, v_{j}\right)+d\left(v_{j}, x^{\prime}\right)$. But now 


$$
\begin{aligned}
d\left(s_{2}, x\right) & =d\left(s_{2}, v_{i}\right)+d\left(v_{i}, x\right) \\
& =d\left(s_{2}, v_{i}\right)+d\left(v_{i}, v_{j}\right)+d\left(v_{j}, x^{\prime}\right) \\
& >d\left(s_{2}, v_{j}\right)+d\left(v_{j}, x^{\prime}\right) \\
& =d\left(s_{2}, x^{\prime}\right) .
\end{aligned}
$$

This implies that $s_{2}$ (and so $S$ ) disitinguishes $x$ and $x^{\prime}$, which is a contradiction.

Claim 3. If $P_{2}\left[v_{j}, v_{i}^{*}\right]$ contains a vertex from $S$ then $P_{2}\left(v_{i}^{*}, v_{i}\right]$ contains no vertex from $S$. The proof is similar as in Claim 3.

Now, we apply the above claims in order to conclude the proof. Notice that if $P_{2}\left[v_{i}, v_{j}^{*}\right]$ contains a vertex from $S$ then due to Claims 2 and 3, all vertices of $S$ are contained in $P\left[v_{j}, v_{j}^{*}\right]$. Since $S$ contains a geodesic triple this is possible only if $g$ is even and $v_{j}$ and $v_{j}^{*}$ belong to $S$. The fact that $v_{j}^{*}$ is element of $S$ implies $d\left(v_{i}, x\right)=d\left(v_{i}, v_{j}\right)+d\left(v_{j}, x^{\prime}\right)$ but then $v_{j} \in S$ distinguishes $x$ and $x^{\prime}$, which is a contradiction. We argue similarly if $P_{2}\left[v_{i}^{*}, v_{j}\right]$ contains a vertex from $S$.

On the other hand if there is no vertex of $S$ in $P_{2}\left[v_{i}, v_{j}^{*}\right]$ and $P_{2}\left[v_{i}^{*}, v_{j}\right]$, then by Claim 1, $S$ can contain at most one vertex in $P_{2}\left(v_{i}^{*}, v_{j}^{*}\right)$ and in $P_{1}\left(v_{i}, v_{j}\right)$ which is a contradiction with the assumption that $S$ is of size at least three.

Now we show the edge version of the previous lemma.

Lemma 5 Let $G$ be a unicyclic graph and let $S$ be a geodesic triple of vertices from $C$. Then, $S$ distinguishes any two edges that does not belong to a same component of $G-E(C)$.

Proof. Suppose to the contrary that $S$ does not distinguish two edges that belong to two distinct components of $G-E(C)$, say $e=x y$ and $e^{\prime}=x^{\prime} y^{\prime}$. If $C$ is of length 3 , then $S$ contains all vertices of $C$, and consequently promptly follows that $S$ disitnguishes $e$ and $e^{\prime}$. So, we assume that $C$ is of length $\geq 4$.

We will consider three cases regarding whether $e, e^{\prime}$ belong to $C$ in order to conlcude the proof.

Suppose first that neither $e$ nor $e^{\prime}$ belongs to $C$. We may assume that $x$ is closer to $C$ than $y$, and similarly $x^{\prime}$ is closer to $C$ than $y^{\prime}$. Then $d(e, s)=d(x, s)$ and $d\left(e^{\prime}, s\right)=d\left(x^{\prime}, s\right)$ for every $s \in S$. Now, Lemma 4 assures that a vertex from $S$ distinguishes $x$ and $x^{\prime}$, and so it distinguishes $e$ and $e^{\prime}$ as well.

Suppose now that both $e$ and $e^{\prime}$ belong to $C$. Let $P_{x}$ and $P_{y}$ be the paths from $C-e-e^{\prime}$. We may assume that end-vertices of $P_{x}$ are $x$ and $x^{\prime}$ and the end-vertices of $P_{y}$ are $y$ and $y^{\prime}$. Notice that a vertex $s$ from $P_{x}$ does not distinguish $e$ and $e^{\prime}$ only if $P_{x}$ is of even length and $s$ sits in the middle of it. Similarly holds for $P_{y}$. But as we have at least three vertices in $S$, we conclude that the third one must distinguish these two edges.

Suppose now that $e$ belongs to $C$ and $e^{\prime}$ does not. We may assume that $x^{\prime}$ is closer to $C$ than $y^{\prime}$. In case $x^{\prime}=x$ note that there is a vertex $s \in S$ such that $d(s, e)=d(s, y)<$ $d(s, x)$, since otherwise $S$ would not contain geodesic triple. But then $d(s, x)=d\left(s, x^{\prime}\right)=$ $d\left(s, e^{\prime}\right)$ further implies $S$ distinguishes $e$ and $e^{\prime}$. Similarly argue when $x^{\prime}=y$. So, we can assume $x^{\prime}$ is distinct from $x$ and $y$. 
Next, consider the graph $G / e$ obtained by contracting the edge $e$ into a vertex $v_{x y}$. As $C$ is of length $\geq 4$, the new graph is also a unicyclic graph. Notice that for any vertex $s$ from $C$, we have $d_{G}(s, e)=d_{G / e}\left(s, v_{x y}\right)$ as the corresponding shortest paths belong to the cycles $C$ and $C / e$, and they coincide.

Now, by Proposition 5, we have a vertex $s$ that distinguishes $x^{\prime}$ and $v_{x y}$ in $G / e$. Consider two possibilities in order to complete the proof. First, if $d_{G}\left(x^{\prime}, s\right)=d_{G / e}\left(x^{\prime}, s\right)$, then the same $s$ distinguishes $x^{\prime}$ and $e$ in $G$, and hence $e^{\prime}$ and $e$. The second possibility is when $d_{G}\left(x^{\prime}, s\right)=d_{G / e}\left(x^{\prime}, s\right)+1$, i.e. the shortest path between $s$ and $x^{\prime}$ goes through edge $e$, and it gets decreases by 1 after contracting $e$. But in that case obviously it must hold $d(s, e)>d\left(s, x^{\prime}\right)=d\left(s, e^{\prime}\right)$ as $x$ and $y$ belong on the shortest path from $s$ to $e^{\prime}$. This concludes the proof.

\section{Branching-resolving sets vs. geodesic triples}

The last two lemmas will now help us to prove that even if a geodesic triple of vertices from the cycle $C$ is not introduced into a branch-resolving set $S$, but there are three $S$-active vertices on that cycle, then the set $S$ will distinguish all pairs of vertices and all pairs of edges. In other words, we will prove that it does not matter if the vertex included in set $S$ is vertex $v_{i}$ from the cycle or any vertex from inside the tree $T_{v_{i}}$, as long as we have a geodesic formation all pairs of vertices and all pairs of edges are distinguished. Let us state and prove this formally.

Lemma 6 Let $G$ be a unicyclic graph and let $S$ be a branch-resolving set of $G$ with $a(S) \geq 3$ and there are three $S$-active vertices on $C$ forming a geodesic triple. Then, $S$ is both a metric generator and an edge metric generator of $G$.

Proof. Let us first prove that $S$ is a metric generator. Suppose to the contrary, i.e. there are two vertices $x, x^{\prime} \in V(G)$ which are not distinguished by $S$. Without loss of generality we may assume that $x \in V\left(T_{v_{0}}\right)$ and $x^{\prime} \in V\left(T_{v_{i}}\right)$ where $i \leq\lfloor g / 2\rfloor$. Since $S$ is a branch-resolving set with $a(S) \geq 3$, Lemma 3 implies $i \neq 0$. Now, Lemma 4 implies that $x$ and $x^{\prime}$ are distinguished by a geodesic triple of $S$-active vertices on $C$, and we want to prove that $x$ and $x^{\prime}$ are distinguished by $S$ as well. Suppose vertices $x$ and $x^{\prime}$ are distinguished by $S$-active vertex $v_{j} \in C$. If $j \notin\{0, i\}$ then for a vertex $s \in S \cap V\left(T_{v_{j}}\right)$ the fact $d\left(x, v_{j}\right) \neq d\left(x^{\prime}, v_{j}\right)$ implies that

$$
d(x, s)=d\left(x, v_{j}\right)+d\left(v_{j}, s\right) \neq d\left(x^{\prime}, v_{j}\right)+d\left(v_{j}, s\right)=d\left(x^{\prime}, s\right)
$$

and the claim follows. Suppose therefore that $j \in\{0, i\}$, say $j=0$. Since $x$ and $x^{\prime}$ are distinguished by $v_{j=0}$, then obviously if $s \in S \cap V\left(T_{v_{0}}\right)$ does not distinguish $x$ and $x^{\prime}$ it must hold $s \neq v_{0}$. Let $P$ be the shortest path connecting vertices $x$ and $s$ and let $v$ be the vertex on path $P$ which is closest to $v_{0}$. Then we have

$$
\begin{aligned}
d(x, s) & =d(x, v)+d(v, s), \\
d\left(x^{\prime}, s\right) & =d\left(x^{\prime}, v_{i}\right)+d\left(v_{i}, v_{0}\right)+d\left(v_{0}, v\right)+d(v, s) .
\end{aligned}
$$


The fact that $d(x, s)=d\left(x^{\prime}, s\right)$ implies

$$
d(x, v)=d\left(x^{\prime}, v_{i}\right)+d\left(v_{i}, v_{0}\right)+d\left(v_{0}, v\right),
$$

where we can add $d\left(v_{0}, v\right)$ to both sides of equality and then from $d\left(v_{0}, v\right) \geq 0$ deduce

$$
d\left(x, v_{0}\right) \geq d\left(x^{\prime}, v_{i}\right)+d\left(v_{i}, v_{0}\right) .
$$

Note that the assumption that three $S$-active vertices on $C$ forming a geodesic triple implies that there must exist an $S$-active vertex $v_{l}$ such that $1 \leq l \leq\lfloor g / 2\rfloor$, so let us denote by $s^{\prime}$ a vertex from $S \cap T_{v_{l}}$. There are two possibilities, it is either $l \leq i$ or $l>i$. In the case when $l \leq i$ we have

$$
\begin{aligned}
d\left(x, s^{\prime}\right) & =d\left(x, v_{0}\right)+d\left(v_{0}, v_{l}\right)+d\left(v_{l}, s^{\prime}\right) \\
& \geq d\left(x^{\prime}, v_{i}\right)+d\left(v_{i}, v_{0}\right)+d\left(v_{0}, v_{l}\right)+d\left(v_{l}, s^{\prime}\right) \\
& >d\left(x^{\prime}, v_{i}\right)+d\left(v_{i}, v_{l}\right)+d\left(v_{l}, s^{\prime}\right) \\
& =d\left(x^{\prime}, s^{\prime}\right),
\end{aligned}
$$

which is a contradiction. In the case when $l>i$ from the facts that $d\left(x, v_{0}\right)>d\left(x^{\prime}, v_{i}\right)$ and $0<i<l \leq\lfloor g / 2\rfloor$ obviously follows $d\left(x, s^{\prime}\right)>d\left(x^{\prime}, s^{\prime}\right)$, which is again a contradiction.

Let us now prove that $S$ is a metric edge generator. Suppose to the contrary, there are two edges $e=x y$ and $e^{\prime}=x^{\prime} y^{\prime}$ which are not distinguished by $S$. We assume that end-vertices of $e$ and $e^{\prime}$ are denoted so that $x$ and $x^{\prime}$ are closer to cycle $C$ then $y$ and $y^{\prime}$ respectively (if there is the difference between those two distances). Again, from $a(S) \geq 2$ and Lemma 5 follows that $e$ and $e^{\prime}$ do not belong to the same connected component of $G-E(C)$.

Suppose first that neither $e$ nor $e^{\prime}$ belong to the cycle $C$. Then let $G_{y}, G_{y^{\prime}}$ and $G_{x}$ be the connected components of $G-\left\{e, e^{\prime}\right\}$ containing vertices $y, y^{\prime}$ and $x$ respectively. If $G_{y}$ or $G_{y^{\prime}}$ contain a vertex from $S$, then $e$ and $e^{\prime}$ would be distinguished by $S$, therefore $S \subseteq V\left(G_{x}\right)$. But for every $z \in V\left(G_{x}\right)$ we have $d(e, z)=d(x, z)$ and $d\left(e^{\prime}, z\right)=d\left(x^{\prime}, z\right)$, so $e$ and $e^{\prime}$ must be distinguished by $S$ since $x$ and $x^{\prime}$ are distinguished by $S$, so we obtained a contradiction.

Suppose now that both $e$ and $e^{\prime}$ belong to the cycle $C$. Lemma 5 implies that $e$ and $e^{\prime}$ are distinguished by $S$-active vertex from $C$, say $v_{k}$. But then $d\left(e, v_{k}\right) \neq d\left(e^{\prime}, v_{k}\right)$ implies that for $s \in S \cap V\left(T_{v_{k}}\right)$ we have

$$
d(e, s)=d\left(e, v_{k}\right)+d\left(v_{k}, s\right) \neq d\left(e^{\prime}, v_{k}\right)+d\left(v_{k}, s\right)=d\left(e^{\prime}, s\right),
$$

which is a contradiction.

Suppose finally that exactly one of $e$ and $e^{\prime}$ is contained on $C$, say $e^{\prime}$. Without loss of generality we may assume $e \in E\left(T_{v_{0}}\right)$ and $e^{\prime}=v_{i} v_{i+1}$ where $0 \leq i \leq\lfloor g / 2\rfloor-1$. Again, Lemma 5 implies that $e$ and $e^{\prime}$ are distinguished by an $S$-active vertex from $C$, say $v_{k}$. If $k \neq 0$ then the similar argument as in previous case yields that $e$ and $e^{\prime}$ must be distinguished by $S$. Suppose therefore that $k=0$. Let $s \in S \cap V\left(T_{0}\right)$, then $s \neq v_{0}$ otherwise $e$ and $e^{\prime}$ would be distinguished by $s$. Since $s$ does not distinguish $e$ and $e^{\prime}$, the fact $s \neq v_{i}$ implies $d\left(e, v_{0}\right)>d\left(e^{\prime}, v_{0}\right)$. Since there is a geodesic triple of $S$-active vertices on $C$, there must exist $S$-active vertex $v_{l}$ for $1 \leq l \leq\lfloor g / 2\rfloor-1$. The fact $d\left(e, v_{0}\right)>d\left(e^{\prime}, v_{0}\right)$ implies $d\left(e, v_{l}\right)>d\left(e^{\prime}, v_{l}\right)$ which further implies $d\left(e, s^{\prime}\right)>d\left(e^{\prime}, s^{\prime}\right)$ for $s^{\prime} \in S \cap V\left(T_{v_{l}}\right)$. Therefore, $e$ and $e^{\prime}$ are distinguished by $S$ which is a contradiction. 


\section{$5 \quad$ Vertex and edge dimensions of unicyclic graphs}

In this section, we show that value of the vertex metric dimension and the value of edge metric dimension of a unicyclic graph can be one of two consecutive integers, whose values are determined by a formula of $L(G)$ and $b(G)$. Recall that $b(G)$ denotes the number of branch-active vertices on the only cycle $C$ in a unicyclic graph $G$. With the use of Lemmas 2 to 6 we obrain easily the following Theorem 7. Later with more involved arguments we extend it to cactus graphs.

Theorem 7 Let $G$ be an unicyclic graph. Then each of $\operatorname{dim}(G)$ and $\operatorname{edim}(G)$ has value $L(G)+\max \{2-b(G), 0\}$ or $L(G)+\max \{2-b(G), 0\}+1$.

Proof. Let $S$ be a vertex or edge metric generator of $G$ of the smallest possible size. Notice that $S$ must contain a set of vertices $S^{*} \subseteq V(G) \backslash V(C)$ that is a branch-resolving set for $G$. As we are assuming that $S$ is a smallest possible set, we may assume that $\left|S^{*}\right|=L(G)$ and in particular $a\left(S^{*}\right)=b(G)$. Lemma 2 implies that at least $\max \{2-b(G), 0\}$ vertices must be introduced to the branch-resolving set $S^{*}$ in order to become a vertex (resp. an edge) metric generator. Therefore, $S \backslash S^{*}$ contains a set $S^{\prime}$ of $\max \{2-b(G), 0\}$ vertices from $C$. Thus, we obtain that $S^{*} \cup S^{\prime}$ must be of order at least $L(G)+\max \{2-b(G), 0\}$. This establishes the lower bound.

Let us now use the result of Lemma 6 to obtain the upper bound. We use the same notation as above. Notice that the set of active vertices of $S^{*}$ union $S^{\prime}$, i.e. $A\left(S^{*}\right) \cup S^{\prime}$ is of order at least 2 but this union may or may not contain a geodesic triple. Notice by introducing to any set of vertices of $C$ of size $\geq 2$ a carefully selected new vertex $x$, we can always assure that the enlarged set $A\left(S^{*}\right) \cup S^{\prime} \cup\{x\}$ contains a geodesic triple. Now Lemma 6 implies that $S^{*} \cup S^{\prime} \cup\{x\}$ is a vertex (resp. an edge) metric generator of $G$. As the latter union is of size $L(G)+\max \{2-b(G), 0\}+1$, we establish the upper bound.

The above theorem gives us promptly the following result.

Corollary 8 Let $G$ be a unicyclic graph. Then $|\operatorname{dim}(G)-\operatorname{edim}(G)| \leq 1$.

\section{Metric dimensions in graphs with edge disjoint cy- cles}

The results for unicyclic graphs from previous sections can now be extended to graphs with more cycles than one, as long as those cycles are edge disjoint. Namely, if cycles in a graph are edge disjoint, then for every cycle in such graph there is a restriction of the graph to a unicyclic subgraph in which that cycle is the only cycle. Such restrictions are not necessarily disjoint, but they cover the whole graph. Then, for a set of vertices in a graph we can also consider a restriction to a unicyclic subgraphs (with few necessary accomodations) and then apply to it the results from previous sections, which then yields the conditions under which such set is a metric generator in a wider graph. In orther to realize all this, we introduce the following more formal definitions. 
We say that $G$ is a cactus graph if all cycles in $G$ are pairwise edge disjoint. Let $C$ be a cycle in a cactus graph $G$ and let $v$ be a vertex on it. Note that the connected component $T_{v}$ of $G-E(C)$ in the case of a cactus graph does not have to be a tree, it can be a cactus graph, i.e. it may contain cycles. Nevertheless, we extend the definition of branching and branch-active vertices in a similar way. Similarly, we denote by $b(C)$ the number of branch-active vertices on $C$ (which now cannot be denoted by $b(G)$ as we did with unicyclic graphs, since cactus graph may have more than one cycle).

Lemma 9 Let $G$ be a cactus graph with c cycles $C_{1}, C_{2}, \ldots, C_{c}$. Then, both $\operatorname{dim}(G)$ and $\operatorname{edim}(G)$ are greater or equal than

$$
L(G)+\sum_{i=1}^{c} \max \left\{2-b\left(C_{i}\right), 0\right\} .
$$

Proof. Let $S \subseteq V(G)$ be a set of vertices from $G$ such that $|S|<L(G)+\sum_{i=1}^{c} \max \{2-$ $\left.b\left(C_{i}\right), 0\right\}$. We want to prove that such $S$ can be neither a vertex nor an edge metric generator. Note that the one of the following must hold for the set $S$ :

- there is a vertex $v$ in $G$ of degree $\geq 3$ such that there are two threads attached to $v$ which do not contain a vertex from $S$;

- there is a cycle $C$ in $G$ such that at most one vertex on $C$ is $S$-active.

In the first case, let $w_{1}$ and $z_{1}$ be the vertices on those two threads incident to $v$, then obviously $w_{1}$ and $z_{1}$ are not distinguished by $S$, therefore $S$ is not a vertex metric generator. The same holds for edges $v w_{1}$ and $v z_{1}$ which means $S$ cannot be an edge metric generator either.

In the second case, if there is no $S$-active vertices on $C$ it means $S=\phi$, so $S$ cannot be a metric generator. If there is exactly one $S$-active vertex $v$ on $C$, let $v_{1}$ and $v_{2}$ be the two neighbors of $v$ on $C$. Then obviously $v_{1}$ and $v_{2}$ are not distinguished by $S$, consequently $S$ cannot be a vertex metric generator. The same holds for edges $v_{1} v$ and $v_{2} v$ and thus $S$ cannot be an edge metric generator either.

We say that a path $P$ in a cactus graph $G$ is a connector of cycles $C_{i}$ and $C_{j}$ of $G$ if the end-vertices $u$ and $v$ of $P$ belong to $V\left(C_{i}\right)$ and $V\left(C_{j}\right)$, respectively, and $P$ does not share any other vertex besides $u$ and $v$ with any cycle in $G$ (for the illustration see Figure 3). We also say that $P$ connects $C_{i}$ and $C_{j}$ or that it is incident to those two cycles. The domain $G_{i}$ of the cycle $C_{i}$ in a cactus graph $G$ is the graph consisting of the cycle $C_{i}$, all connector paths incident to $C_{i}$ and all threads and branches incident to either $C_{i}$ or the corresponding connector paths (see Figure 4). Note that $G_{i}$ is a unicyclic graph with $C_{i}$ being its only cycle. Also, note that for two distinct cycles $C_{i}$ and $C_{j}$ their corresponding domain graphs $G_{i}$ and $G_{j}$ may not be vertex disjoint because there may exist a connector path between cycles $C_{i}$ and $C_{j}$ in which case their corresponding domains $G_{i}$ and $G_{j}$ share that connector paths and all threads and branching hanging on the vertices of that connector. Finally, $G_{i}$ is obviously an isometric subgraph of $G$. 
We say that a vertex $v_{j}$ from a domain $G_{i}$ is a boundary vertex if $v_{j}$ belongs to $C_{j}$ in $G$ where $j \neq i$. Note that the boundary vertex $v_{j}$ is actually an end-vertex of the connector path between cycles $C_{i}$ and $C_{j}$. We say that a vertex $v \in V(G) \backslash V\left(G_{i}\right)$ is on the other side of the boundary vertex $v_{j}$ of $G_{i}$ if the shortest path from $v$ to $C_{i}$ contains $v_{j}$ (see again Figure 4).

So far we have established domains in a cactus graph which are unicyclic graphs and which cover the whole $G$. Since we are interested in sets of vertices $S$ in a cactus graph which are metric generators, we also want to divide such sets into parts which will be metric generators in those domains, but we cannot simply take a restriction of the set $S$ onto a domain, since two vertices or two edges from the same domain can in a cactus graph be distinguished by a vertex $s \in S$ which is outside that domain, because of which we must add boundary vertices to the restriction. Therefore, for a set of vertices $S$ in $G$, let $S_{i}$ denote the set obtained from $S \cap V\left(G_{i}\right)$ by adding all boundary vertices from the domain $G_{i}$ to it. Observe that the following holds: if $S$ is a branch-resolving set in $G$ then $S_{i}$ is a branch-resolving set in $G_{i}$, also if there is a geodesic triple of $S$-active vertices on every cycle in $G$ then there is a geodesic triple of $S_{i}$-active vertices on $C_{i}$ in $G_{i}$.

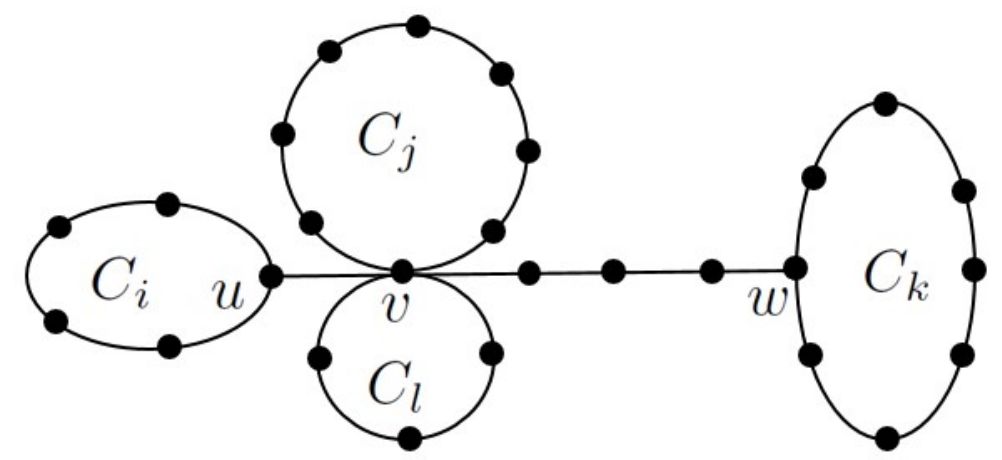

Figure 3: An illustration of a connector path: the edge $u v$ is a connector of cycles $C_{i}$ and $C_{j}$ and also of $C_{i}$ and $C_{l}$. The path from $v$ to $w$ is a connector of cycles $C_{j}$ and $C_{k}$, but the path from $u$ to $w$ is not a connector of cycles $C_{i}$ and $C_{k}$. Note that a connector path can consist of only one vertex ( $v$ is a connector of $C_{j}$ and $C_{l}$ ).

Lemma 10 Let $G$ be a cactus graph with c cycles $C_{1}, C_{2}, \ldots, C_{c}$. Then, both $\operatorname{dim}(G)$ and $\operatorname{edim}(G)$ are smaller or equal than

$$
L(G)+\sum_{i=1}^{c} \max \left\{2-b\left(C_{i}\right), 0\right\}+c .
$$

Proof. Let us construct a set of vertices $S$ such that $|S|$ is smaller or equal than (2), for which we will prove it is both a vertex and an edge metric generator. The set $S$ will consist of three parts as the desired bound is the sum of three numbers. Let $S_{a}$ be a minimum branch-resolving set in $G$, thus $\left|S_{a}\right|=L(G)$. Further, let $S_{b}$ consist of $\max \left\{2-b\left(C_{i}\right), 0\right\}$ vertices from every cycle $C_{i}$ not contained in $S_{a}$. Note that $S_{b}$ contains 


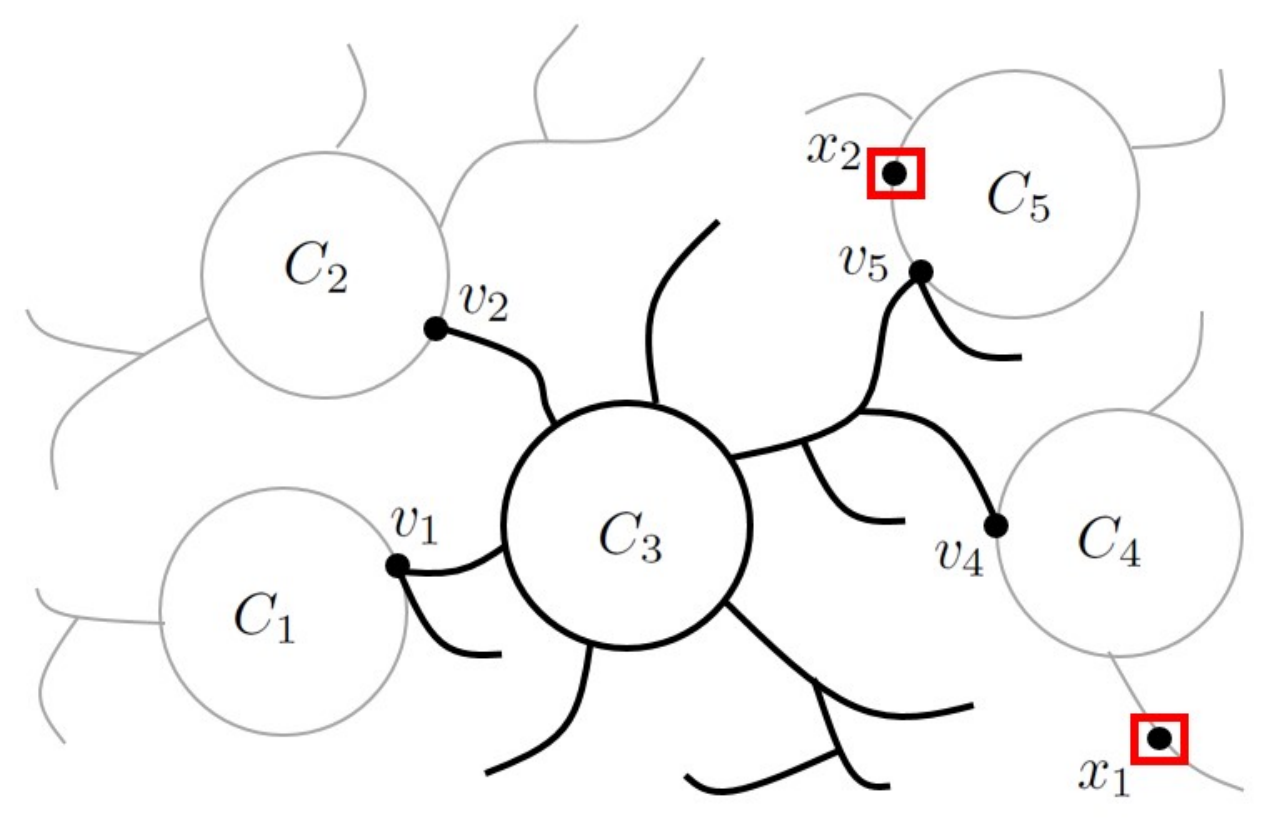

Figure 4: An example of a cactus graph with five cycles where the domain $G_{3}$ of the cycle $C_{3}$ is emphesized. Vertices $v_{1}, v_{2}, v_{4}$ and $v_{5}$ are the boundary vertices of the domain $G_{3}$. With respect to the domain $G_{3}$, the vertex $x_{1}$ is on the other side of the boundary vertex $v_{4}$, while $x_{2}$ is on the other side of the boundary vertex $v_{5}$.

at most two vertices from every cycle in $G$ which are chosen so that every cycle in $G$ will have at least two $\left(S_{a} \cup S_{b}\right)$-active vertices. Finally, note that a cycle in $G$ may or may not have a geodesic triple of $\left(S_{a} \cup S_{b}\right)$-active vertices, so we define a third set $S_{c}$ in a following way. Let $S_{c}$ contain a vertex from every cycle in $G$ which does not have a geodesic triple of $\left(S_{a} \cup S_{b}\right)$-active vertices, which vertex is chosen so that it forms a geodesic triple with the two $\left(S_{a} \cup S_{b}\right)$-active vertices which must exist on each cycle. Notice again that for any two vertices on a cycle we can choose easily the third one so that they form a geodesic triple. Therefore, $\left|S_{c}\right| \leq c$. Now we define $S=S_{a} \cup S_{b} \cup S_{c}$. Obviously

$$
|S| \leq L(G)+\sum_{i=1}^{c} \max \left\{2-b\left(C_{i}\right), 0\right\}+c .
$$

Also, note that $S$ is a branch-resolving set with a geodesic triple of $S$-active vertices on every cycle $C_{i}$ in $G$.

We want to prove that $S$ is both a vertex and an edge metric generator. Let $x$ and $x^{\prime}$ be any two distinct vertices or two edges in $G$. In order to do so, we distinguish the following two cases.

Case 1: $x, x^{\prime}$ both belong to a same domain. Denote this domain by $G_{i}$. Since $S_{i}$ is a branch-resolving set with a geodesic triple of $S_{i}$-active vertices in a unicyclic graph $G_{i}$, then Lemma 6 implies $S_{i}$ distinguishes $x$ and $x^{\prime}$ in $G_{i}$. Recall that $G_{i}$ is a isometric subgraph of $G$, so if $x$ and $x^{\prime}$ are distinguished by a vertex $s \in S_{i} \cap S$ then $x$ and $x^{\prime}$ are 
distinguished by $S$ in $G$. Suppose now that $x$ and $x^{\prime}$ are distinguished in $G_{i}$ by $s \in S_{i} \backslash S$. This implies that $s$ is a boundary vertex in $G_{i}$, but this further implies there must exist a vertex $s_{1} \in S$ on the other side of the boundary vertex $s$ since otherwise we would have a contradiction with our assumption that there are at least two $S$-active vertices on every cycle in $G$. Now, as $s$ distinguishes $x$ and $x^{\prime}$ then certainly $s_{1} \in S$ distinguishes them too, so $x$ and $x^{\prime}$ are distinguished by $S$ in $G$.

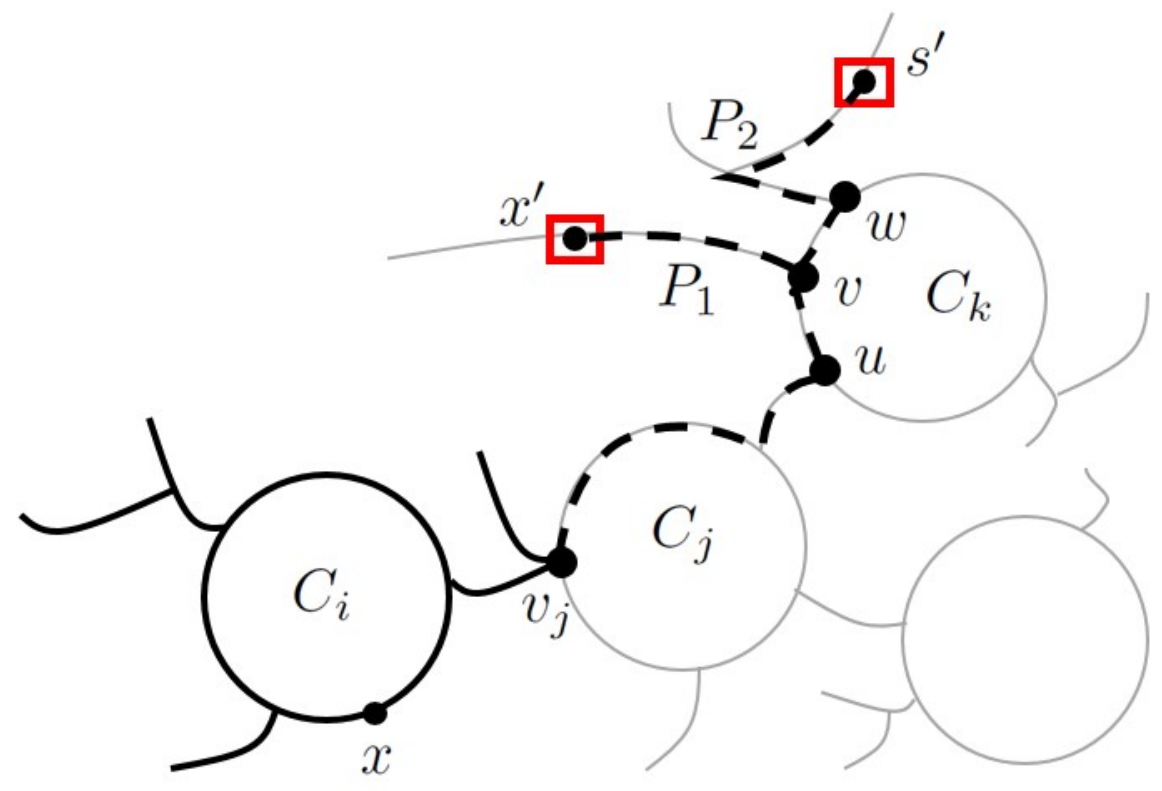

Figure 5: An illustration of the graph $G_{i}^{\prime}$ : the domain $G_{i}$ of the cycle $C_{i}$ contains $x$, but does not contain $x^{\prime}$ nor $s^{\prime}$, so it has to be extended by paths $P_{1}$ and $P_{2}$ which lead from the boundary vertex $v_{j}$ to $x^{\prime}$ and $s^{\prime}$ respectively.

Case 2: $x, x^{\prime}$ do not belong to a same domain. Suppose that $x$ belongs to $G_{i}$ and $x^{\prime}$ belongs to $G_{k}$ where $k \neq i$. So, we assume that $x^{\prime}$ does not belong to $G_{i}$ and $x$ does not belong to $G_{k}$, otherwise it reduces to the previous case.

In this case we also want to apply the result for the unicyclic graphs, but as $x$ and $x^{\prime}$ do not belong to a same domain, now we will have to expand the domain $G_{i}$ which contains $x$ so that it contains $x^{\prime}$ as well. In order to do so (and the construction that follows is illustrated in Figure 5), let $v_{j} \in V\left(C_{j}\right)$, for $j \neq i$, be the boundary vertex of the domain $G_{i}$ closest to $x^{\prime}$. Let $u$ be the vertex on cycle $C_{k}$ closest to $v_{j}$ and let $v$ be the vertex on the cycle $C_{k}$ closest to $x^{\prime}$. Finally, assuming that $l_{k}$ denotes the length of the cycle $C_{k}$, let $w$ be the $S$-active vertex on $C_{k}$ such that vertices $u, v$ and $w$ belong to a same half of the cycle $C_{k}$, where a half of the cycle is any path on the cycle of length $\leq\left\lceil\left(l_{k}-1\right) / 2\right\rceil$. Note that it is always possible to choose such $w$ because there is a geodesic triple of $S$-active vertices on the cycle $C_{k}$.

Let $P_{1}$ be the shortest path from $x^{\prime}$ to $v_{j}$ in $G$, note that $P_{1}$ contains the vertex $v$. Let $s^{\prime} \in S$ be a vertex not contained in the domain $G_{i}$ on the same side of the boundary 
vertex $v_{j}$ as $x^{\prime}$, which is chosen so that the shortest path $P_{2}$ from $s^{\prime}$ to $v_{j}$ contains the vertex $w$. This is possible since $w$ is $S$-active vertex. Finally, let $G_{i}^{\prime}$ be the extension of the domain $G_{i}$ obtained by adding paths $P_{1}$ and $P_{2}$ to it. Note that the extension $G_{i}^{\prime}$ is also a unicyclic graph with $C_{i}$ being its only cycle. Also, note that the distances in $G_{i}^{\prime}$ and $G$ are the same, i.e. $G_{i}^{\prime}$ is a isometric subgraph of $G$, due to the way in which we chose vertices $u, v$ and $w$.

Let $z$ be the common vertex of paths $P_{1}$ and $P_{2}$ furthest from $v_{j}$ in $G_{i}^{\prime}$. The construction of $G_{i}^{\prime}$ implies $z=v$ or $z=w$. Note that the addition of paths $P_{1}$ and $P_{2}$ to $G_{i}$ in order to obtain $G_{i}^{\prime}$ certainly creates two threads hanging at $z$ in $G_{i}^{\prime}$ which do not contain a vertex from $S_{i}$. Therefore, the set $S_{i}$ is not a branch-resolving set in $G_{i}^{\prime}$, so it is not a good candidate for metric generator. Hence, just as we modified the domain $G_{i}$ into $G_{i}^{\prime}$, now the set $S_{i}$ also has to be modified into $S_{i}^{\prime}$. We define $S_{i}^{\prime}=S_{i} \backslash\left\{v_{j}\right\} \cup\left\{s^{\prime}\right\}$, i.e. we replace the boundary vertex $v_{j}$ with $s^{\prime}$ in the set $S_{i}$ to obtain $S_{i}^{\prime}$. Note that the set $S_{i}^{\prime}$ defined in this way certainly is a branch-resolving set in $G_{i}^{\prime}$, because exchanging the vertex $v_{j}$ with $s^{\prime}$ in $S_{i}$ resolves the problem of two threads hanging at $z$ in $G_{i}^{\prime}$. Also, note that there must exist a geodesic triple of $S_{i}^{\prime}$-active vertices on $C_{i}$ in $G_{i}^{\prime}$. Thus, according to Lemma 6. so $S_{i}^{\prime}$ distinguishes $x$ and $x^{\prime}$ in $G_{i}^{\prime}$. Since $G_{i}^{\prime}$ is isometric subgraph of $G$, this further implies $S$ distinguishes $x$ and $x^{\prime}$ in $G$ and the case is proven.

The above two lemmas give us promptly the following result.

Corollary 11 Let $G$ be a cactus graph with c cycles. Then $|\operatorname{dim}(G)-\operatorname{edim}(G)| \leq c$.

The bound $c$ in the above Corollary 11 is the best possible as the graphs for which the both sides of the bound are achieved are presented in [8].

\section{Further work}

In the paper, we have established that the vertex and edge metric dimensions of a unicyclic graph differ by at most 1 , more precisely each of them has its value in

$$
L(G)+\max (2-b(G), 0) \quad \text { and } / \text { or } \quad L(G)+\max (2-b(G), 0)+1 .
$$

A possible further work is to research (maybe characterize) when each of these two values is realized for each of $\operatorname{dim}(G)$ and $\operatorname{edim}(G)$. Our ongoing investigation shows, beside maybe other things, that it depends also of the parity of $C$. Let us mention that this will fully resolve when the difference of $\operatorname{dim}(G)-\operatorname{edim}(G)$ is $-1,0$ and 1 .

Problem 12 For a unicyclic graph $G$, determine when the difference $\operatorname{dim}(G)-\operatorname{edim}(G)$ is $-1,0$ and 1 .

One can propose even a more general problem as follows.

Problem 13 For a cactus graph $G$ with c cycles, determine when the difference $\operatorname{dim}(G)-$ $\operatorname{edim}(G)$ is $-c,-c+1, \ldots,-1,0,1,2, \ldots, c-1, c$. 
Another direction is to consider graphs with higher cyclomatic number, which is defined as $c(G)=|E(G)|-|V(G)|+1$, where $n=|V(G)|$ and $m=|E(G)|$. Regarding trees have cyclomatic number 0 , unicyclic graphs have 1 , and other graphs have $\geq 2$. Note that for trees we have $|\operatorname{dim}(G)-\operatorname{edim}(G)|=0$, unless $G$ is $K_{2}$. Similarly holds for unicyclic graphs by Corollary 8 . So maybe this relation can be generalized to graphs with higher cyclomatic number, in particular we believe the following holds.

Conjecture 14 Let $G \neq K_{2}$ be a graph with cyclomatic number c. Then

$$
|\operatorname{dim}(G)-\operatorname{edim}(G)| \leq c
$$

Note that the above conjecture holds for more dense graphs, in particular for graphs with cyclomatic number $c \geq n-1$ as $n-1 \geq|\operatorname{dim}(G)-\operatorname{edim}(G)|$ for every $G$. Also note that if the bound of the conjecture holds, then it is tight, see [8] for infinitely many graphs with $c$ vertex-disjoint cycles each. Moreover there are presented graphs with $\operatorname{dim}(G)-\operatorname{edim}(G)=c$ as well graphs with $\operatorname{edim}(G)-\operatorname{dim}(G)=c$.

Acknowledgements. The authors acknowledge partial support Slovenian research agency ARRS program P1-0383 and ARRS project J1-1692 and also Project KK.01.1.1.02.0027, a project co-financed by the Croatian Government and the European Union through the European Regional Development Fund - the Competitiveness and Cohesion Operational Programme.

\section{References}

[1] L. M. Blumenthal, Theory and applications of distance geometry, Oxford University Press, Oxford (1953).

[2] J. Caceres, C. Hernando, M. Mora, I. M. Pelayo, M. L. Puertas, C. Seara, D. R. Wood, On the metric dimension of Cartesian products of graphs, SIAM J. Discrete Math. 21 (2) (2007) 423-441.

[3] F. Harary, R. A. Melter, On the metric dimension of a graph, Ars Combin. 2 (1976) 191-195.

[4] A. Kelenc, Distance-Based in Variants and Measures in Graphs, PhD thesis, University of Maribor, Faculty of Natural Sciences and Mathematics, 2020.

[5] A. Kelenc, N. Tratnik, I. G. Yero, Uniquely identifying the edges of a graph: the edge metric dimension, Discrete Appl. Math. 251 (2018) 204-220.

[6] A. Kelenc, D. Kuziak, A. Taranenko, I. G. Yero, Mixed metric dimension of graphs, Appl. Math. Comput. 314 (1) (2017) 429-438.

[7] S. Khuller, B. Raghavachari, A. Rosenfeld, Landmarks in graphs, Discrete Appl. Math. 70 (1996) 217-229. 
[8] M. Knor, S. Majstorović, A. T. Masa Toshi, R. Škrekovski, I. G. Yero, Graphs with the edge metric dimension smaller than the metric dimension, arXiv:2006.11772 [math.CO]:21 Jun 2020.

[9] I. Peterin, I. G. Yero, Edge metric dimension of some graph operations, Bull. Malays. Math. Sci. Soc. 43 (2020) 2465-2477.

[10] A. Sebő, E. Tannier, On metric generators of graphs, Math. Oper. Res. 29 (2) (2004) 383-393.

[11] P. J. Slater, Leaves of trees, Congr. Numer. 14 (1975) 549-559.

[12] Y. Zhang, S. Gao, On the edge metric dimension of convex polytopes and its related graphs, J. Comb. Optim. 39 (2) (2020) 334-350.

[13] E. Zhu, A. Taranenko, Z. Shao, J. Xu, On graphs with the maximum edge metric dimension, Discrete Appl. Math. 257 (2019) 317-324.

[14] N. Zubrilina, On the edge dimension of a graph, Discrete Math. 341 (7) (2018) 2083-2088. 